\title{
Tempo sensitivity in auditory sequences: Evidence for a multiple-look model
}

\author{
CAROLYN DRAKE and MARIE-CLAIRE BOTTE \\ Université René Descartes, Paris, France
}

\begin{abstract}
Differential thresholds for tempi (with interonset intervals ranging from 100 to $1,500 \mathrm{msec}$ ) were measured using an adaptive 2IFC paradigm for several types of auditory sequences. In Experiment 1, the number of intervals in an isochronous sequence was varied to compare the sensitivity for single intervals with that for sequences of two to six intervals. Mean relative just noticeable differences (JNDs) decreased as the number of intervals increased (single intervals $=6 \%$, two intervals $=4 \%$, four intervals $=3.2 \%$, six intervals $=3 \%$ ) and were optimal at intermediate tempi for both sequences and single intervals (as low as $1.5 \%$ in the range between 300 and $800 \mathrm{msec}$ ). In Experiment 2, the sensitivity for different types of irregular sequences was studied. Globally, JNDs for irregular sequences were of an intermediate level between that observed for single intervals and that observed for regular sequences. However, the closer a sequence was to regularity, the lower its relative JND. Experiment 3 demonstrated that musicians were more sensitive than nonmusicians to changes in tempo, and this was true for single intervals and for regular and irregular sequences, demonstrating the role of training on these abilities. The results are discussed in terms of possible underlying mechanisms, in particular those providing a mental representation of the mean and dispersion of successive interval durations.
\end{abstract}

How small a change in duration are we able to detect? The psychoacoustical literature on time perception suggests that when subjects are asked to compare two intervals (empty or filled by a sound) they are able to say which is longer or shorter when there is a difference of at least $6 \%$ to $10 \%$ of the standard duration (Abel, 1972; Allen, 1979; Creelman, 1962; Getty, 1975, 1976; Small \& Campbell, 1962; Woodrow, 1951). This is true within a range of $200-2,000 \mathrm{msec}$, and the actual precision depends on many different factors, in particular the method used and the physical characteristics of the events marking the intervals. A similar level of sensitivity is also found when subjects are asked to detect a change in duration of one or two of the intervals contained in regular and rhythmic sequences (Drake, 1990, 1992, 1993; Drake, Botte, \& Gérard, 1989; Drake, Gérard, \& Botte, in press; Halpern \& Darwin, 1982; Hirsh, Monahan, Grant, \& Singh, 1990; Monahan \& Hirsh, 1990; van Noorden, 1975). However, when subjects are asked to compare the rate or tempo of two isochronous sequences, the little data available suggests that subjects are much more sensitive to changes, since they are able to detect a change of about 2\% (Michon, 1964).

How can we explain this greater sensitivity to changes in tempo of sequences than to changes in duration of single intervals? An obvious answer is that in the case of

We wish to thank Professors Douglas Creelman, Ken Grant, and Peter Killeen for their helpful reviews and Mari Jones, Jean Lorenceau, and Steve McAdams for their comments on previous versions of this manuscript. Correspondence should be addressed to $\mathrm{C}$. Drake, Laboratoire de Psychologie Expérimentale, CNRS URA 316, 28 nue Serpente, 75006 Paris, France. isochronous sequences, listeners are able to extract multiple cues-one from each interval in each sequence, whereas only a single cue is available in the case of single intervals. But what is the relationship between the processing involved in the two cases? There are at least two possible theoretical positions. The first considers that there are two distinct mechanisms: one for judging the duration of single intervals and one for judging the tempo of sequences. The second position considers that the same underlying mechanism is involved in the two cases, but that the functioning is more elaborate in longer sequences. Partial support for a single mechanism was provided by Fraisse (1963), who found evidence suggesting that the same patterns of sensitivity are found for single intervals and sequences. For single intervals, he describes data indicating that, contrary to Weber's law, which predicts that relative just noticeable differences (JNDs) are proportional to the stimulus duration, sensitivity is maximum in a relatively narrow range of interonset intervals (IOIs) centered around $600 \mathrm{msec}$. For sequences, when subjects were asked to discriminate between regular and irregular sequences, Fraisse (1967) also found a zone of maximum sensitivity around 600 -msec IOIs. However, contradictory evidence was provided by Michon (1964), who presented data indicating that the zone of maximum sensitivity for changes in tempo of sequences is considerably faster (about 100-msec IOIs) than that found by Fraisse for single intervals ( $600 \mathrm{msec})$. Michon's pattern of tempo JND suggests that, on the contrary, two different mechanisms are functioning. The series of three experiments presented here examined these two possibilities and provides support for the position of a single mechanism. 
How exactly is the information concerning interval duration used? How is the listener able to extract an accurate memory trace of the first stimulus and compare it successfully with the second? We propose to summarize the processes in the following way: When asked to discriminate the durations of single intervals, listeners must create a memory trace of the first interval which they compare with their memory trace of the second interval. However, we suggest that when listeners are asked to discriminate the tempi of two sequences, they do not simply repeat this comparison as many times as there are intervals in the sequence (a first strategy, which we will refer to as "interval-by-interval comparison"), but rather that they abstract information concerning the relationship between each interval in the sequence, probably on the basis of the mean and dispersion of the interval durations (a second strategy, which we will refer to as "multiple look"). The repetition of regular intervals would allow for "multiple looks" of the same interval, thus increasing the precision of the trace as a function of the number of "looks." The values of JND, considered as the standard deviation of the response distribution, would then decrease inversely to the square root of the number of intervals in the sequence (Green \& Swets, 1973). Whatever the system, it would necessarily be limited in the range of tempi at which it could function.

The first aim of this paper was to confirm that processing of tempo changes in sequences is indeed better than processing changes in duration of single intervals, by examining the two situations under the same experimental conditions. In Experiment 1, relative JNDs were measured for changes in tempo in regular sequences containing a varying number of intervals over a wide range of tempi. Subjects heard a first sequence followed by a second sequence that was identical in all ways except that it was slightly faster or slightly slower in tempo. Their task was to say which sequence was faster. Differential thresholds for tempo are taken as an indication of the ease or sharpness of processing: under conditions of optimal processing, we expect the lowest thresholds. If additional processing is occurring in sequences compared with that occurring in single intervals, then a greater sensitivity to changes in tempo should be seen for sequences than for single intervals. Also, since the amount of available information increases with the number of intervals, JNDs should decrease as the number of intervals increases.

The second question examined whether a similar pattern of JNDs in relation to the tempo tested is observed for single intervals and sequences, a way of testing between one or two mechanisms. Is the form of the expected $\mathrm{U}$-shaped curve the same in both single intervals and sequences, or is the zone of maximum sensitivity shifted towards more rapid tempi in the processing of sequences as Michon's data would suggest?

A third question considered whether the lower JNDs for sequences result from a process of multiple look rather than from a process of interval-by-interval comparison. This was done by examining whether or not overall time discriminations produced by regular sequences are finer than those produced by irregular ones. In Experiment 2, relative JNDs for changes in tempo were measured for irregular sequences which varied in their proximity to regularity. Subjects heard the same irregular sequence played twice at slightly different tempi and were asked to indicate which of the two was slower. The comparison of JNDs for these irregular sequences with those for regular sequences from Experiment 1 should thus clarify the mode of functioning: If listeners simply remember the duration of each interval in the first sequence and compare it with the duration of each interval in a comparison sequence, then no difference should be found between JNDs for regular and irregular sequences containing the same number of events and having the same total durations. On the other hand, if listeners extract and use information about the mean and dispersion of the interval durations in the sequence, sensitivity to tempo changes should be considerably greater in regular than in irregular sequences, due to the lower interval-duration dispersion in the former.

A fourth question, that of the role of training in temporal regularity on the tempo JND for different types of sequences, was investigated in Experiment 3 by comparing the results of nonmusician subjects with those of musicians who had explicitly learned to focus on the temporal structure of sound sequences and also to adjust their motor responses to what they hear. The evolution of sensitivity was also examined over four repetitions. For these two factors (subjects and repetitions), we focused in particular on whether similar improvements are seen for single intervals and regular and irregular sequences.

To summarize, we investigated whether the ability to judge the duration of single intervals and the ability to detect changes in tempo of sequences stem from a single mechanism or whether they involve two distinct mechanisms. The aim of the three experiments presented here was to throw light on this question and on the mode of the functioning of such mechanisms by testing how well different groups of subjects were able to detect changes in the tempo of sequences varying in length and temporal structure.

\section{EXPERIMENT 1 \\ Optimal Tempo Sensitivity for Single Intervals and Isochronous Sequences}

This first experiment asked three questions. First, are the differential thresholds found for isochronous sequences the same as those for single intervals? Second, do the relative JNDs decrease as the number of intervals in a sequence increases? Third, is the same U-shaped curve with a zone of maximum sensitivity around $600 \mathrm{msec}$ found to be the same for sequences and single intervals?

\section{Method}

Subjects. Four subjects, all members of the Auditory Perception Department, participated in Experiment 1. They were all familiar 
with this type of psychoacoustic experiment, and all had normal hearing.

Materials. Four types of monotonic sequences varying in the number of intervals they contained $(1,2,4$, or 6$)$ were constructed, and each was presented at 11 different standard tempi, ranging from very fast $(100$-msec IOI) to very slow $(1,500$-msec IOI); 100-, 200-, 300-, 400-, 500-, 600-, 700-, 800-, 900-, 1,000-, 1,500-msec IOIs). Although tempo is traditionally expressed by the number of events per minute, we adopted the inverse, that is, the duration between successive onsets (IOI) for ease of presentation and comparison with other data. Sequences were composed of $440-\mathrm{Hz}, 50-\mathrm{msec}$ tones, which were presented binaurally at $70 \mathrm{~dB}$ SPL, with off-periods depending on the tested tempo. The two sequences in a trial were separated by an interval twice the IOI of the first sequence in order to maintain regularity between the two sequences.

Apparatus. Signals were digitally generated in real time by a synthesizer (DMX 1000) and controlled by a microcomputer (Northstar Horizon). The subject sat in a soundproof room and listened via earphones (TDH 49).

Procedure. The subjects heard one sequence followed by another which was slightly different in tempo, and their task was to indicate, by pressing one of two keys, which sequence was faster. The variable tempo was initially set to be slower than the standard. An adaptive procedure developed by Levitt (1971) was used, wherein the response given by the subject determined the values of the following stimuli: two successive correct answers resulted in a decrease of $1 \%$ in the tempo difference between the two sequences, one incorrect answer led to an increase of $1 \%$. Differential thesholds were calculated on the last 10 (of 12) reversals. This two-interval forced-choice paradigm converges on a $70.7 \%$ probability of correct detections. Some advantages of this procedure over traditional psychophysical methods are its robustness, small-sample reliability, and avoidance of floor and ceiling effects. Each experimental session comprised the JND measurements of the 11 different tempi for one of the four types of sequences $(1,2,4$, or 6 intervals). Each JND measurement was repeated three times. The measurement of JND for one stimulus took from 8 to $15 \mathrm{~min}$, depending on the tempo and number of events in the stimulus. Thus, subjects completed 12 sessions of about $2 \mathrm{~h}$ each spread over no more than 3 weeks. Orders were counterbalanced within and between sessions.

\section{Results}

Only the JNDs obtained for the last two repetitions were included in the analysis, with the first being considered a practice run. As is usual in studies of duration and tempo discrimination, the dependent measure was the relative rather than the absolute JND. This means that the results are presented in percentage of the standard tempo under study rather than in milliseconds. Figure 1 presents the mean relative JNDs obtained for the 11 standard tempi for the single intervals and the 2-, 4-, and 6-interval sequences. This figure also includes curves for theoretical predictions that are analyzed in the General Discussion. An analysis of variance (ANOVA) was run on the relative JNDs by number of intervals $(1,2,4$, and 6$)$, tempo (100-, 200-, 300-, 400-, 500-, 600-, 700-, 800-, 900-, 1,000-, and 1,500-msec IOI), and repetition (second and third).

The first question concerns the general level of these thresholds for the single intervals and the sequences. A planned comparison between these two types of stimuli revealed a significant difference $[F(1,3)=115.4, p<$ $.01]$. For the single intervals, the mean relative JNDs were

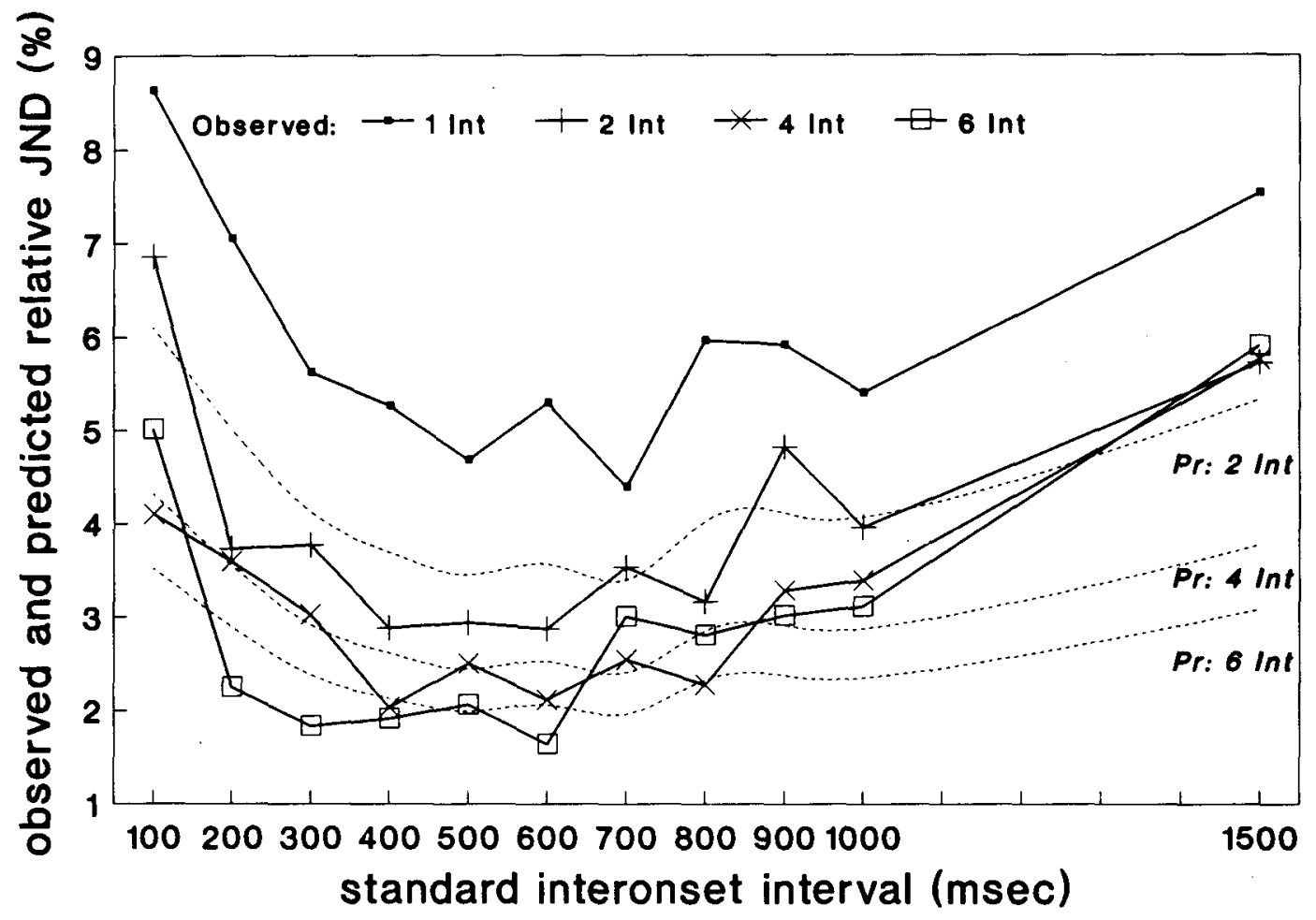

Figure 1. Experiment 1: Mean relative JND for standard tempi ranging from 100- to 1,500-msec IOls for sequences containing 2,3,5, and 7 tones. Data are averaged over two repetitions and 4 subjects. Theoretical predictions for the multiple-look mechanism are added (dotted curves). 
$6 \%$ with a range of $4.4 \%-8.6 \%$, slightly lower than those noted previously. However, for the sequences, the average value was $3.4 \%$, with a range of $1.6 \%-6.9 \%$. The lowest values were below $2 \%$, which means that subjects were able to detect a change in tempo of $6 \mathrm{msec}$ for sequences with $300-\mathrm{msec}$ IOI, and a change of $12 \mathrm{msec}$ for sequences with $600-\mathrm{msec}$ IOI. The standard deviations ranged from $1.5 \%$ for medium tempi to $2.6 \%$ for fast and slow tempi.

The second question asked whether sensitivity increased as the number of intervals in the sequences increased. The same ANOVA also revealed a main effect of number of events $[F(3,9)=70.0, p<.01]$, with mean relative JND falling as the number of events in the sequence increased ( 1 interval $=6 \%, 2$ intervals $=4 \%, 4$ intervals $=3.2 \%$, 6 intervals $=3 \%$ ). Planned comparisons revealed a significant difference between sequences with 1 and 2 intervals $[F(1,3)=52.3, p<.01]$ and between sequences with 2 and 4 intervals $[F(1,3)=64.3, p<.01]$, but no significant differences were found between 4 and 6 intervals.

The third question considered whether the same pattern of JNDs was found over the range of tempi examined for the two types of stimuli. The same ANOVA revealed a main effect of tempo $[F(9,27)=16.2, p<.01]$. The zone of optimum sensitivity observed here between 300 and $800 \mathrm{msec}$ was wider than that described by Fraisse's work. If two distinct mechanisms exist, we may see an interaction between tempo and number of events, with tempo having a different effect for single intervals than for sequences of 2, 4, and 6 intervals. Figure 1 indicates that the four curves have similar shapes, and this lack of effect is confirmed by a planned comparison which revealed no significant interaction between these two factors. A distinct pattern, however, is seen for the very slow tempi $(1,500$-msec IOI), where the JND is lower for sequences than for single intervals but no additional improvements are seen as the number of intervals in the sequence increases. These data throw light on the factor limiting the additional processing in sequences: the maximum temporal window within which it occurs may be about $3,000 \mathrm{msec}$ (two 1,500-msec intervals). This value may determine the maximal number of intervals providing an improvement of JND at a given tempo. An additional point is that the maximum sensitivity for sequences is found here for tempi considerably slower than those found by Michon (1964). These two points will be examined in the General Discussion below.

It could be argued that subjects were not basing their judgments on changes in duration of the intervals in the sequences but rather on the total duration of the sequence. However, the JNDs provided a clear indication that this was not the case. For example, the sequence with four 300 -msec intervals had a total duration of $1,200 \mathrm{msec}$. Therefore, if subjects were basing their judgments on the total duration of the sequence, JND would be equivalent to that for the single duration of $1,200 \mathrm{msec}$, which is above 6\% (see Figure 1). However, the observed relative JND in these sequences was about $3 \%$.

\section{Discussion}

This experiment provides three main results. First, differential thresholds were lower for sequences than for single intervals, suggesting that some additional processing is involved in sequences relative to single intervals. Second, as the number of intervals in the sequence increased, the efficiency of the system improved, suggesting that the additional processing is related to the amount of information available to the listener as provided by the number of intervals in the sequence. Third, contrary to the hypothesis of two distinct mechanisms, a similar pattern of JNDs as a function of tempo was observed for both single intervals and sequences, with no interaction between the two factors. Thus, while additional processing appears to occur for sequences, there is no evidence of a mechanism distinct from that involved in single intervals.

\section{EXPERIMENT 2 \\ Tempo Sensitivity for Irregular Sequences}

The results of Experiment 1 indicate that additional processing may indeed be occurring for sequences relative to single intervals. However, it does not provide direct support for the claim that such a mechanism would be related to the extraction of the mean and dispersion of the intervals in the sequence (multiple look). This question was addressed in Experiment 2 by examining whether regular sequences were processed better than irregular ones. We measured relative JND for different types of irregular sequences to see whether or not these values were higher than those observed for comparable regular sequences with the same number of intervals and total duration. If a multiple-look mechanism is involved, the irregular sequences would be expected to lead to higher differential thresholds than the regular sequences, whereas if an interval-by-interval-comparison mechanism is involved, no difference should be seen between the two types of sequences, since they contain the same number of intervals (and therefore comparisons). There may also be a continuum of difficulty among the irregular sequences, depending on their deviation from regularity. In addition, some sequences may have distinctive patterns of increasing or decreasing interval durations that make them easier to discriminate.

\section{Method}

Subjects. The 4 subjects used in Experiment 1 also took part in Experiment 2.

Materials. All the irregular sequences contained four intervals, the sum of which was always equal to $2,000 \mathrm{msec}$. They were therefore comparable to the Experiment 1 condition with 4 intervals of $500 \mathrm{msec}$. This condition was chosen because of the systematically low observed thresholds. In Experiment 2, each IOI was slightly modified away from 500 msec to make the sequence irregular: each successive interval was either $15 \%$ longer or $15 \%$ shorter than the preceding interval, so each interval was easily discriminable from the adjacent intervals. There were three possible locations for changes of IOI within a sequence (between Intervals 1 and 2, 2 and 3 , or 3 and 4 ). Figure 2 presents the three groups of irregular sequences that were defined on the basis of the pattern of increases 
and decreases: sequences in Group 1 had alternating increases and decreases, those in Group 2 had two increases or decreases in succession, those in Group 3 had three increases or decreases in succession. Sequences in Group 3 had a special status, since they constituted the progressive acceleration or deceleration in tempo that is often found in music, speech, and motor activity. Are subjects able to abstract this second-degree regularity and use it in the tempo-discrimination task? The three groups of sequences can also be distinguished on two other criteria indicated in Figure 2. First, a measure of the dispersion of the different IOIs that they contain is calculated by taking the standard deviation of the absolute differences between each IOI used and the mean value of $500 \mathrm{msec}$. These values are given in the "sd" column of Figure 2 . Sequences in Group 1 have much lower standard deviations, indicating that although each individual interval is as easily discriminable from the preceding and following intervals as it is in other sequences $( \pm 15 \%)$, the particular pattern of intervals results in sequences much closer, on average, to a regular sequence of $500 \mathrm{msec}$. A second means of describing the sequences is to consider the range of intervals obtained by subtracting the shortest interval in the sequence from the longest interval. This value is given in the "range" column of Figure 2. Group 1 sequences have the narrowest range, and Group 3 sequences have the widest range. In order to respect the constraints presented above ( $15 \%$ increases and decreases, and constant total sequence duration), the IOIs in Figure 2 were the only ones possible.

Apparatus. The apparatus was the same as that used in Experiment 1 .

Procedure. The procedure was the same as that used in Experiment 1 , with the eight sequences presented in a counterbalanced order.

\section{Results}

Figure 3 presents the mean relative JND for each of the eight irregular sequences. An ANOVA on the relative JND for the eight sequences and two repetitions revealed no significant main effects. Planned comparisons on the

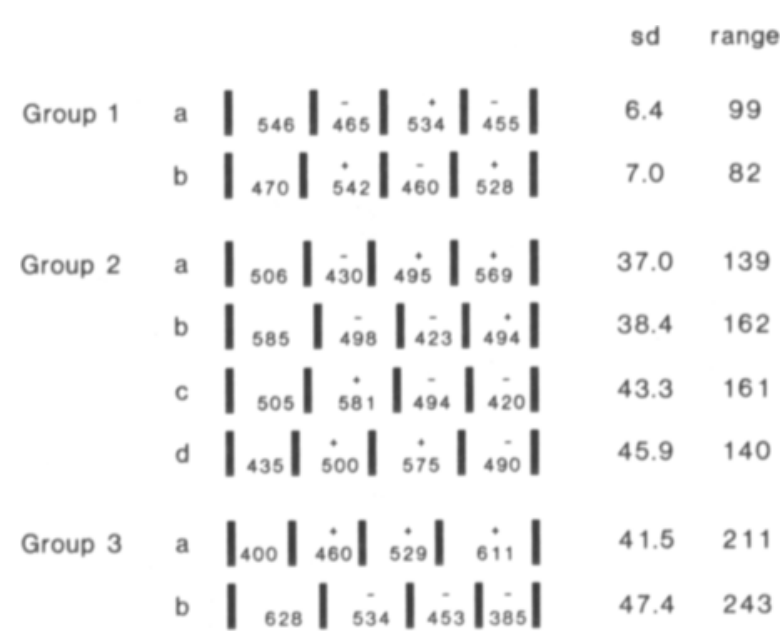

Figure 2. Experiment 2: Eight irregular sequences used as stimuli separated into three groups on the basis of the number of successive increases or decreases in IOI. Bars representing events are separated by $10 I$ values in milliseconds; "+" and "_" stand for either a $15 \%$ lenghthening or a $15 \%$ shortening of the current IOI relative to the preceding one. On the right of the figure are the mean standard deviations of absolute differences between the IOIs and 500 msec ("sd"), and the total range of IOIs ("range") in milliseconds.

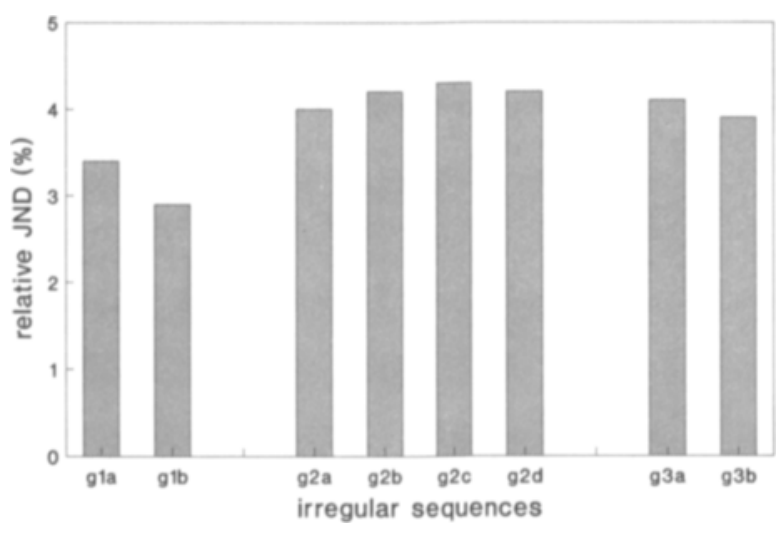

Figure 3. Experiment 2: Mean relative JNDs for 8 irregular sequences of 5 tones with 500 msec between successive onsets. Data averaged over two trials and 4 subjects.

results for the three types of sequences, however, revealed that Group 1 sequences ( $\mathrm{gla}$ and glb) had significantly lower JNDs $(3.2 \%)$ than the other types of sequences $(4.1 \%)[F(1,3)=9.6, p<.01]$, and that there was no significant difference between Group 2 and Group 3. The standard deviations ranged from $1.5 \%$ to $2.7 \%$ and were not systematically related to the groups of irregular sequences.

These results are interesting when they are compared with the mean relative JND obtained in Experiment 1 for regular sequences and single intervals. Figure 4 shows the mean relative JND obtained in Experiment 2 for the three types of irregular sequences based around $500 \mathrm{msec}$. It also shows those obtained in Experiment 1 for regular sequences with four 500 -msec intervals and for those obtained for single intervals of $500 \mathrm{msec}$. The considerable difference between the results for the regular sequences and for the single interval has been interpreted as an indication that additional processing occurs in the former. Therefore, if the irregular sequences also involve this additional processing, the mean relative JND should be close to that observed for regular sequences, whereas if such a mechanism is not functional, the results should be closer to those observed for the single intervals. Figure 4 shows that mean relative JNDs for the three groups of irregular sequences are intermediate between those observed for the regular sequences and those observed for the single intervals. Planned comparisons indicate no significant difference between the JND for regular sequences and the JND for the Group 1 irregular sequences, and similarly no significant difference between the single intervals and the Group 2 and Group 3 irregular sequences.

\section{Discussion}

This experiment provides support for the hypothesis of a process of multiple-look rather than of interval-byinterval comparision, since the JNDs observed were higher for the irregular than for regular sequences. It also suggests that the multiple-look strategy can be extended 


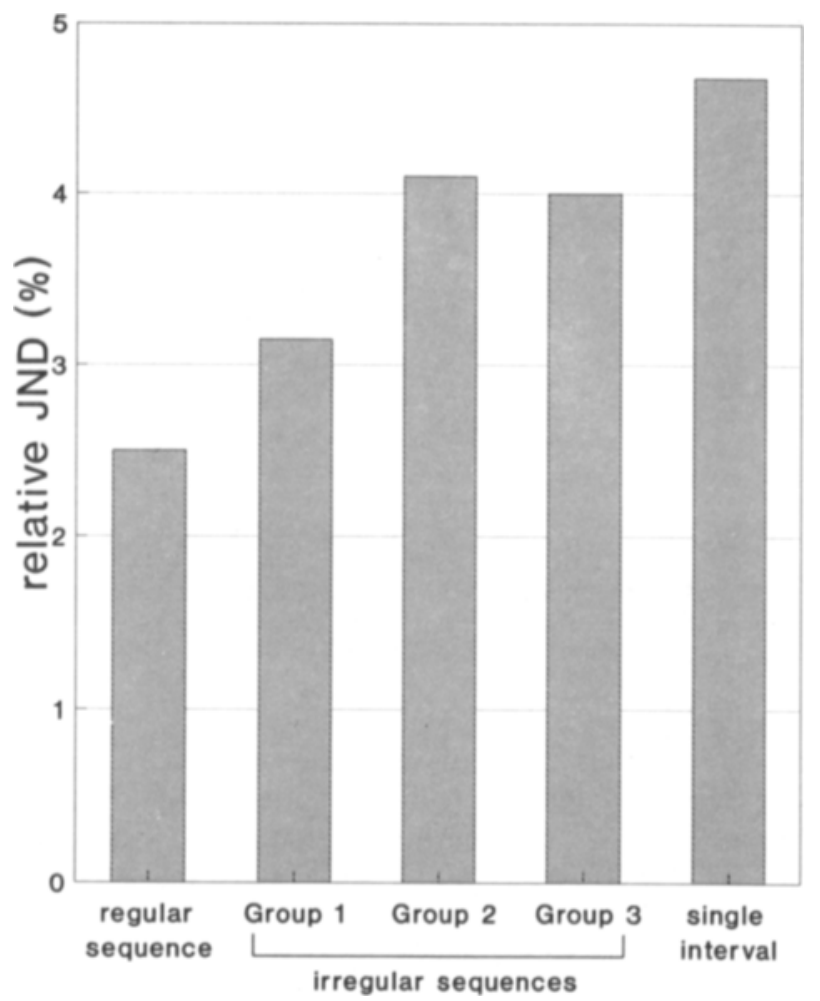

Figure 4. Experiments 1 and 2: Mean relative JNDs for the single durations, regular sequences, and three types of irregular sequence. Data averaged over two trials and 4 subjects.

to irregular sequences, which can be separated into two types: those with a low interval standard deviation (Group 1), for which the JNDs are close to those obtained for regular sequences, and those with a high interval standard deviation (Groups 2 and 3), for which the JNDs are close to those obtained for single intervals. Thus, although the Group 1 irregular sequences are not truly regular, they could still be processed efficiently: because of the low level of interval dispersion, the memorized trace of one sequence is easily discriminable from that of a sequence slightly slower or faster. Also, the accelerating and decelerating sequences (Group 3) did not lead to low relative JNDs, suggesting that this second-order regularity is not useful for tempo discrimination. These results also indicate that the mean standard deviation rather than the range of IOI is the pertinent factor in determining the accuracy of detection of changes in tempo.

\section{EXPERIMENT 3}

\section{Influence of Musical Training on Tempo Sensitivity}

The role of training in temporal regularity on the ability to detect changes in tempo of regular and irregular sequences was examined in Experiment 3 by comparing the performance of nonmusician subjects with that of musicians, presuming the latter to have learned explicitly to focus on the temporal structure of sound sequences, in particular regular ones, as well as to adjust their motor responses to what they are hearing. We were also interested in the evolution of sensitivity over four repetitions of each experimental condition. Key conditions were selected from Experiments 1 and 2. In Experiment 3, we compared relative JNDs for four types of sequences: a single interval, a regular sequence, and two irregular sequences (one with a low standard deviation, the other with a high standard deviation as defined in Experiment 2). The results of Experiments 1 and 2 had suggested that the mechanism allowing for the creation of a memory trace of a single interval is also involved in the processing of tempo in sequences-probably due to some type of multiple-look mechanism providing a more accurate representation of the interval duration. It therefore seemed reasonable to expect that the ability to detect changes in tempo would be more affected by musical training than would the ability to detect changes in the duration of single intervals.

\section{Method}

Subjects. Two groups of 7 subjects took part in this experiment: 7 nonmusicians who had never received any musical training and 7 musicians who had received at least 5 years of musical training and who played an instrument almost every day.

Materials. Four sequences were chosen from Experiments 1 and 2: single intervals, regular sequences with five tones, and two irregular sequences of five tones (sequence $A=g 3 a$ and sequence $\mathrm{B}=\mathrm{g} 1 \mathrm{~b}$ from Experiment 2). The range of tempi examined was reduced: a fast tempo of $300 \mathrm{msec}$ at the lower limit of the maximum sensitiviy zone, a medium tempo of $600 \mathrm{msec}$ at the middle point, and a slow tempo of $900 \mathrm{msec}$ at the upper limit of the same zone.

Apparatus. The apparatus was the same as that used in Experiments 1 and 2.

Procedure. A slightly varied procedure was used in this third experiment in order to shorten the duration of the experiment: instead of the rule used previously requiring two correct answers before a downward step change and one incorrect answer for an upward step change (two down/one up), we adopted a new rule of four down/one up, which converged on detection values of $84.1 \%$. Stable results were obtained more quickly by this stricter criterion; 6 reversals sufficed (rather than the 12 used previously) and the experimental time was reduced by about one half. Of course, threshold values obtained here were necessarily higher than those of Experiments 1 and 2, but the pattern of results remained comparable. In addition, subjects were asked to indicate which of the two sequences was the slower (as opposed to the faster in Experiments 1 and 2). A pilot study had shown that there were no differences in these two procedures.

\section{Results}

Figure 5 presents the mean relative JND for the nonmusicians and the musicians for the four types of sequences and the three tempi examined. An ANOVA was carried out on the mean relative JNDs for 2 subject groups (musicians and nonmusicians), 4 types of sequence (single interval, regular sequence, and irregular sequences $A$ and B), 3 tempi $(300,600$, and $900 \mathrm{msec})$, and 4 repetitions. Overall, the musicians had lower JNDs $(6.2 \%)$ than the nonmusicians $(8.8 \%)[F(1,12)=17.6, p<.01]$. As in Experiments 1 and 2, lowest JNDs were observed for the 


\section{nonmusicians}
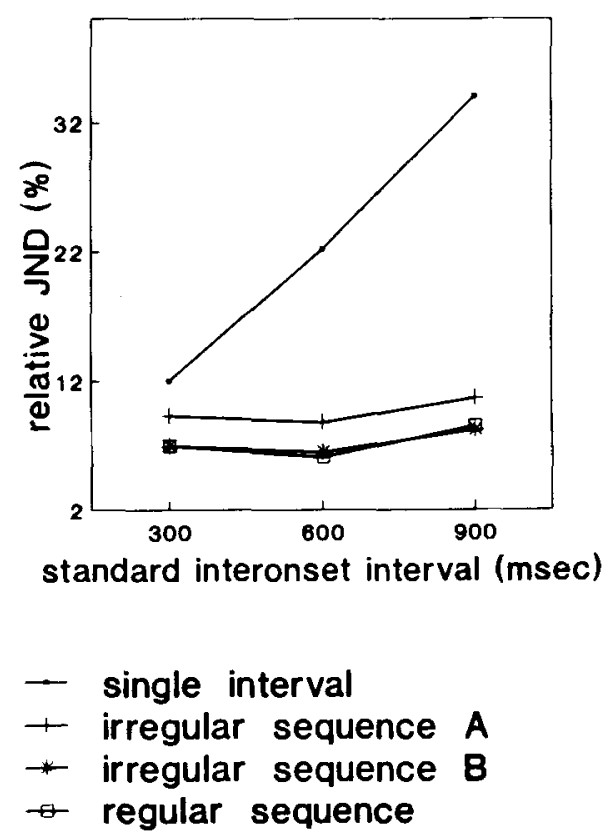

\section{musicians}

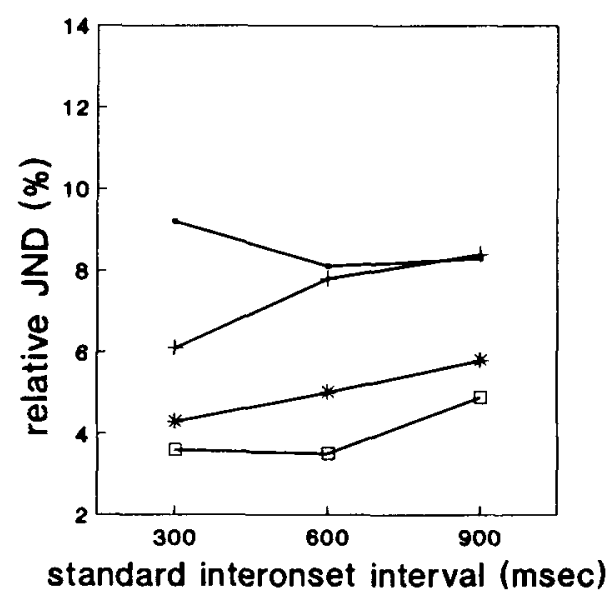

Figure 5. Experiment 3: Mean relative JNDs for the two groups of subjects (musicians and nonmusicians), the four types of sequence (single interval, regular sequence, and irregular sequences $A$ and B), and the three tempi (300-, 600-, and 900-msec IOI).

isochronous sequence $(5.6 \%)$, intermediate values were found for the irregular sequences (high dispersion $\mathrm{A}=$ $8.5 \%$, low dispersion $B=6.1 \%$ ), and the highest JNDs were seen for the single interval $(9.9 \%)[F(3,36)=72.2$, $p<.01]$. As in Experiment 1, there was a significant effect of tempo $[F(2,24)=25, p<.01]$, with lowest JND for the intermediate tempo $(600 \mathrm{msec}=7 \%)$ and higher JND for the faster $(300 \mathrm{msec}=7.3 \%)$ and slower sequences $(900 \mathrm{msec}=\mathbf{8 . 3 \%})$. Performance also im- proved over repetitions $[8.5 \%, 7.5 \%, 7.3 \%$, and $6.8 \%$; $F(3,36)=24.6, p<.01]$. The range of standard deviations was the same as that seen previously and did not vary systematically between the group of subjects or type of sequence. Thus, the patterns of results found in this experiment were very similar to those found in Experiments 1 and 2.

In accordance with the hypothesis of a single mechanism, no interaction was observed between the group of subjects and the types of sequence, indicating that musical training results in a general ability of finer temporal discriminations whatever the type of sequence used. However, the two groups of subjects did differ in their zones of optimum sensitivity. Figure 6 shows a significant interaction between subject group and tempo $[F(2,24)=6.2$, $p<.01]$, with two equally low mean JNDs observed at the fastest and medium tempi for the musicians, but with a single minimum JND for the medium tempo for the nonmusicians. This means that the zone of optimal sensitivity extends to tempi that are faster for musicians than for nonmusicians. However, Figure 6 shows that this tendency is seen only for the three types of sequences and not for the single intervals. Moreover, although the musicians perform better than the nonmusicians, a similar training effect with repetitions is seen in both cases (no significant interaction between subject group and repetitions).

\section{Discussion}

While confirming the preceding results for nonmusicians, the results of Experiment 3 highlight significant differences between the two groups of subjects. The musicians were able to detect much smaller changes in tempo between two sequences than were the nonmusicians (as low as $11 \mathrm{msec}$ in regular sequences with a tempo of $300 \mathrm{msec}$-see Figure 6). They also appear to have a wider zone extending towards faster tempi in which they are extremely sensitive to changes in tempo, a result which deserves to be confirmed with a wider range of tempi. The fact that the group of subjects and tempo had the same effect for the four different types of sequence supports

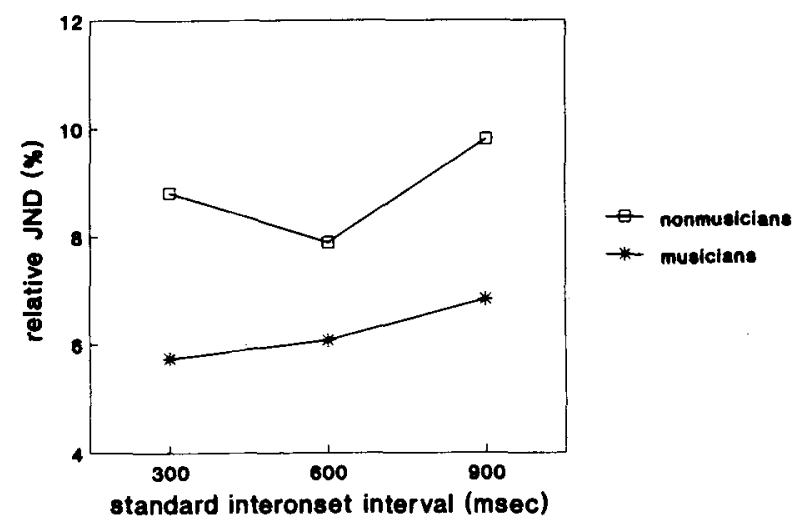

Figure 6. Experiment 3: Mean relative JNDs for the two groups of subjects (musicians and nonmusicians) and the three tempi (300-. 600-, and 900-msec IOI). 
the hypothesis of a single mechanism and indicates that musical training leads to general improvements in both single intervals and sequences.

\section{GENERAL DISCUSSION}

Studies in time perception have traditionally concentrated on how well listeners are able to detect changes in the duration of single intervals; more recently they have concentrated on how these abilities are modified when single intervals are incorporated into sequences of auditory events. In both cases, changes as low as $6 \%$ to $10 \%$ of the duration of the interval in question are detected. In this paper, we have examined a particular instance of the ability to judge the duration of intervals which perhaps has more relevance in everyday life: that of the rate or tempo of a sequence. The principle result is that listeners are much more sensitive to changes in tempo of regular sequences than they are to changes in tempo of many irregular sequences and to changes in the duration of single intervals.

\section{One Mechanism Rather Than Two}

The observation that listeners are more sensitive to changes in tempo in sequences than they are to changes in duration of single intervals could suggest that two distinct mechanisms are involved. However, the fact that similar patterns of tempo sensitivity are observed for single intervals and for sequences that vary in their number of intervals (Experiment 1) and the fact that this pattern is almost unaffected by the musical experience of subjects (Experiment 3) have led us to reject the hypothesis of two distinct mechanisms in favor of one single mechanism which is used more efficiently in sequences.

\section{Implications for the Modeling of Successive Time Interval Processing}

How could this additional processing function? We have considered two possible strategies-multiple interval-byinterval comparison and multiple-look. The multiple interval-by-interval comparison strategy involves the comparison of the duration of each interval in the first sequence with the duration of the corresponding interval in the second sequence. As discussed previously, Experiments 2 and 3 do not provide support for this hypothesis, since sensitivity to some of the irregular sequences was almost as poor as it was for the single intervals. In addition, such a mechanism would also be extremely onerous and could not be applied to sequences that continued over any length of time, since it would be impossible to maintain the memory trace of an interval in the first sequence until the appropriate position in the second sequence was reached.

The multiple-look strategy probably involves the creation of a memory trace of the average duration and the degree of dispersion of the intervals in the first sequence heard by the subject. The intervals in the second sequence would be compared with this "average" memory trace. Thus, the more intervals in the first sequence, the more precise would be its memory trace and the greater would be the sensitivity: For a single interval, the trace would be elaborated from a single input, and as the number of intervals in a sequence increased, the number of inputs would increase and the trace could become more precise. This strategy is congruent with the results of Experiments 2 and 3, where sensitivity for irregular sequences was intermediate between that for regular sequences and that for single intervals: A similar elaboration of an average memory trace would occur with irregular sequences, but would be less precise due to the higher degree of interval dispersion, resulting in decreased accuracy in the comparison of the two sequences; the irregular sequences would be analyzed in a similar way, but less efficiently.

A quantitative test of the multiple-look model is possible under the hypothesis of independent observations: The observed absolute JNDs (in milliseconds) for single durations obtained in $70.7 \%$ of the cases are converted into absolute JNDs expected in $84.1 \%$ of the cases ( $1 S D$ of $z$-score distribution). These values, obtained for single intervals, are then used to calculate predicted values for sequences of 2,4 , and 6 intervals with the following equation:

$$
\mathrm{JND}_{N}=\mathrm{JND}_{1}(1 / \sqrt{ } N)
$$

where $N$ is the number of intervals. These predicted JNDs, expressed in percentages, are plotted in Figure 1, together with the experimental data. For the sake of clarity, the curves for the predicted JNDs have been smoothed. Overall, the predicted and observed values can be considered to be in the same range, with some notable deviations depending on the range of tempi: For the fastest tempi (100-600-msec IOI), observed JNDs are generally lower than predicted (except at the 100-msec IOI); for intermediate tempi (700-800-msec IOI), observed and predicted values are relatively close; and for slower tempi $(900-$ 1,500-msec IOI), observed JNDs are increasingly higher than the predictions. These comparisons suggest that the simple multiple-look mechanism operates in a limited range of IOI (assuming that the psychometric function is the same for the whole range of tempi).

These deviations from the values predicted by a simple multiple-look strategy suggest two additional hypotheses which are currently under investigation. First, as tempi become slower, the observed JNDs are progressively higher than the predictions, a fact which could be explained by the hypothesis of a process operating within a limited temporal window: once the sequence duration exceeds the span of this temporal window, the process of multiple looks is limited, and JNDs no longer decrease as the number of intervals (or looks) increases. Let us assume that the window within which the multiple looks can be integrated is exactly $3,000 \mathrm{msec}$, probably an oversimplification. Therefore, the maximum number of pos- 
sible looks can be calculated for each combination of IOIs and number of intervals. Under this hypothesis, at 1,500msec IOI the 2-, 4-, and 6-interval conditions allow for only two looks, and so JNDs $\left(\mathrm{JND}_{2}, \mathrm{JND}_{4}, \mathrm{JND}_{6}\right.$ ) should be identical for the three conditions; this is observed. At 900- and 1,000-msec IOI, the 4- and 6-interval conditions are limited to three looks; as predicted, these conditions provide equal JNDs which are better than those for the 2 -interval conditions. However, $\mathrm{JND}_{4}$ and $\mathrm{JND}_{6}$ are not as good as expected because $\mathrm{JND}_{1}$ is divided by only $1 / \sqrt{ } 3$ (3 looks). From 800- to 600-msec IOI, the results are more ambiguous, but they still follow the multiple-look predictions at 500-msec IOI for the 4- and 6-interval conditions.

Second, concerning the fast tempi, the lower than predicted JNDs suggest that another mechanism, in addition to that of the multiple look, may be involved. We are all the more convinced that some additional processing may be involved as the total duration of the sequences at these fast tempi was well below the limits of the temporal window established in the preceding paragraph. For IOIs shorter than $500 \mathrm{msec}$, JNDs are often better than predicted in spite of a window which is more or less filled (for instance, six intervals at $200-\mathrm{msec}$ IOI last only $1,200 \mathrm{msec}$ ). Therefore, it can be assumed that the multiple-look strategy was probably underused in our experimental conditions at these fast tempi. This fact may account for the considerably lower JNDs for tempo observed by Michon (1964): Although it is not stated clearly in his paper, it would appear that Michon used pulse trains of equal total duration rather than of the equal number of intervals used here. Thus, at the faster tempi, Michon's subjects may have heard many more events than did our subjects. Indeed, for the faster tempi, we may not have reached a maximum level of sensitivity with six intervals, and even lower JNDs may be observed with sequences containing more intervals. Nevertheless, the duration of the temporal window, and consequently the number of "useful" intervals, might also depend on the interval duration, a matter currently under investigation.

So, while previous researchers talked about a U-shaped curve of sensitivity with a maximum of about $600 \mathrm{msec}$, a more appropriate description may consist of a curve with three zones: (1) a zone of optimal sensitivity ranging from 300- to 900-msec IOI, depending on the number of intervals in the sequence, which fits the predictions of the multiple-look mechanism relatively well, (2) a zone of lesser sensitivity at slow tempi with JNDs above the predictions, which may be related to the limits of a temporal window, and (3) a zone of greater sensitivity at faster tempi with JNDs below the predictions, which may be related to the use of additional processing's improving the results of a simple multiple-look mechanism.

Let us now look at the way musical training affects the processing of changes in tempo. Overall, musicians have lower JNDs than do nonmusicians, which could be related either to improvements in general abilities in accomplish- ing the task or to a wider temporal window that would allow for more processing time in all conditions. Only one specific change in pattern of results beyond this greater general sensitivity was observed between the two groups of subjects: the zone of maximum sensitivity extended to faster tempi for the musicians for all three types of sequences but not for the single intervals, which suggests that musical training may have a specific effect on the additional processing that could occur at the faster tempi.

A final point of interest is the fact that some irregular sequences which are close to regularity (Group 1) result in JNDs that are almost as low as those observed for truly regular sequences. As long as the dispersion of the inter$\mathrm{val}$ durations is low, irregular sequences appear to be processed in a manner similar to that of regular sequences. The advantage of such a system can clearly be seen in the perception and performance of music, where one of the first organizational principles is the detection of the underlying beat, thus defining the tempo. It is now well established by music performance studies that musicans never perform the temporal structure in a "perfectly regular" fashion, but rather perform with both intentional and unintentional variability (see Drake \& Palmer, 1993, for a review). If the system of extracting the underlying tempo was not flexible, such a process would be impossible in the case of live music, a completely inconceivable position.

\section{REFERENCES}

ABEL, S. M. (1972). Discrimination of temporal gaps. Journal of the Acoustical Society of America, 52, 519-524.

Allen, L. G. (1979). The perception of time. Perception \& Pyschophysics, 26, 340-354.

CreelmaN, C. D. (1962). Human discrimination of auditory duration Journal of the Acoustical Society of America, 34, 582-593.

Drake, C. (1990). Processus perceptifs et cognitifs impliqués dans l'organisation du rythme musical. Unpublished doctoral dissertation, University of Paris V

DrAKE, C. (1992). Les accents musicaux: Y a-t-il une explication perceptive? Journal de Physique IV, 2, 205-208.

DRAKE, C. (1993). Perceptual and performed accents in musical sequences. Bulletin of the Psychonomic Society, 31, 107-110.

Drake, C., Botte, M. C., \& Gérard, C. (1989). A perceptual distortion in simple musical thythms. Proceedings of the Intemational Society of Psychophysics: Fifth Annual Meeting, Cassis, France.

Drake, C., GÉrard, C., \& BotTe, M.-C. (in press). Rhythm perception: Interactions between time and intensity. Contemporary Music Review.

Drake, C., Palmer, C. (1993). Accent structures in music performance. Music Perception, 10, 343-378.

Fraisse, P. (1963). Psychology of time. New York: Harper.

Fraisse, P. (1967). Le seuil différentiel de durée dans une suite régulière d'intervalles. Année Psychologique, 1, 43-49.

GETTY, D. J. (1975). Discrimination of short temporal intervals: A comparison of two models. Perception \& Psychophysics, 18, 1-8.

GetTY, D. J. (1976). Counting processes in human timing. Perception \& Psychophysics, 20, 191-197.

GrEen, D. M., \& SwETS, J. A. (1973). Signal detection theory and psychophysics. Huntington, NY: Krieger.

HALPERN, A. R., DARWIN, C. J. (1982). Duration discrimination in a series of rhythmic events. Perception \& Psychophysics, 31, 86-89. 
Hirsh, I. J., Monahan, C. B., Grant, K. W., \& Singh, P. G. (1990). Studies in auditory timing: 1. Simple patterns. Perception \& Psychophysics, 47, 215-226.

LEVITT, H. (1971). Transformed up-down methods in psychoacoustics. Journal of the Acoustical Society of America, 49, 467-477.

Michon, J. A. (1964). Studies on subjective duration: I. Differential sensitivity in the perception of repeating temporal sequences. Acta Psychologica, 22, 441-450.

Monahan, C. B., \& HiRsh, I. J. (1990). Studies in auditory timing: 2. Rhythm patterns. Perception \& Psychophysics, 47, 227-242.
Small, A. M., \& Campbell, R. A. (1962). Temporal differential sensitivity for auditory stimuli. American Journal of Psychology, 75, 401-410.

VAN NOORDEN, L. (1975). Temporal coherence in the perception of tone sequences. Doctoral thesis: Eindhoven.

Woodrow, H. (1951). Time perception. In S. S. Stevens (Ed.), Handbook of experimental psychology (pp. 1224-1236). New York: Wiley.

(Manuscript received September 14, 1992; revision accepted for publication February 19, 1993.) 